\title{
On classical capacity of Weyl channels
}

\author{
G.G.Amosov*1 \\ ${ }^{1}$ Steklov Mathematical Institute of Russian Academy of \\ Sciences
}

November 3, 2020

\begin{abstract}
The additivity of minimal output entropy is proved for the Weyl channel obtained by the deformation of a q-c Weyl channel. The classical capacity of channel is calculated.
\end{abstract}

Keywords: quantum Weyl channel, classical capacity of a channel

\section{Introduction}

The quantum coding theorem proved independently by A.S. Holevo [1] and B. Schumacher, M.D. Westmoreland 2] posed the task of calculating the Holevo upper bound $\bar{C}\left(\Phi^{\otimes N}\right)$ for a tensor product of $\mathrm{N}$ copies of quantum channel $\Phi$ because a classical capacity of $\Phi$ is given by the formula

$$
C(\Phi)=\lim _{N \rightarrow+\infty} \frac{\bar{C}\left(\Phi^{\otimes N}\right)}{N} .
$$

The additivity conjecture asks whether the equality

$$
\bar{C}(\Phi \otimes \Omega)=\bar{C}(\Phi)+\bar{C}(\Omega)
$$

holds true for the fixed channel $\Phi$ and an arbitrary channel $\Omega$. If the additivity property (11) takes place for $\Phi$ the classical capacity can be calculated as follows

$$
C(\Phi)=\bar{C}(\Phi)
$$

\footnotetext{
*This work is supported by the Russian Science Foundation under grant N 19-11-00086.
} 
The same level of interest has the additivity conjecture in the weak form asking whether

$$
\bar{C}\left(\Phi^{\otimes N}\right)=N \bar{C}(\Phi)
$$

takes place for a fixed channel $\Phi$. The validity of this statement also leads to (2). The additivity conjecture for $\bar{C}$ is closely related to the additivity conjecture for the minimal output entropy of a channel and the multiplicativity conjectures for trace norms of a channel [3]. At the moment, the additivity is proved for many significant cases [4 8] including the solution to the famous problem of Gaussian optimizers [9, 10]. On the other hand, there are channels for which the additivity conjecture doesn't hold true [11]. Recently the method of majorization was introduced to estimate the Holevo upper bound for Weyl channels [12]. In the present paper we prove the additivity conjecture for one subclass of Weyl channels that are "deformations" of q-c channels of [1]. Our method is based upon [12].

Throughout this paper we denote $\mathfrak{S}(H)$ the set of positive unit-trace operators (quantum states) in a Hilbert space $H, I_{H}$ is the identity operator in $H$ and $S(\rho)=-\operatorname{Tr}(\rho \log \rho)$ is the von Neumann entropy of $\rho \in \mathfrak{S}(H)$. Quantum channel $\Phi: \mathfrak{S}(H) \rightarrow \mathfrak{S}(K)$ is a completely positive trace preserving map between the algebras of all bounded operators $B(H)$ and $B(K)$ in Hilbert spaces $H$ and $K$ respectively. Given two $\rho, \sigma \in \mathfrak{S}(H)$ for which supp $\rho \subset$ supp $\sigma$ the quantum relative entropy is $S(\rho \| \sigma)=\operatorname{Tr}(\rho \log \rho)-\operatorname{Tr}(\rho \log \sigma)$. The property of non-increasing the relative entropy with respect to the action of a quantum channel $\Phi$ states [13]

$$
S(\Phi(\rho) \| \Phi(\sigma)) \leq S(\rho \| \sigma)
$$

for $\rho, \sigma \in \mathfrak{S}(H)$.

The Holevo upper bound for a quantum channel $\Phi$ is determined by the formula

$$
\bar{C}(\Phi)=\sup _{\pi_{j}, \rho_{j} \in \mathfrak{S}(H)}\left(S\left(\sum_{j} \pi_{j} \Phi\left(\rho_{j}\right)\right)-\sum_{j} \pi_{j} S\left(\Phi\left(\rho_{j}\right)\right)\right)
$$

where the supremum is taken over all probability distributions $\left(\pi_{j}\right)$ on the ensemble of states $\rho_{j} \in \mathfrak{S}(H)$.

\section{Weyl channels}

Here we use the techniques introduced in [14, 15] and developed in [16 19]. Fix an orthonormal basis $\left(e_{j}, j \in \mathbb{Z}_{n}\right)$ in a Hilbert space $H$ with dimension $\operatorname{dim} H=n$, and consider two unitary operators in $H$ defined by the formula

$$
U e_{j}=e^{\frac{2 \pi i}{n} j} e_{j}, V e_{j}=e_{j+1}, j \in \mathbb{Z}_{n} .
$$


Formula (3) determines unitaries $W_{j k}=U^{j} V^{k}$ called Weyl operators satisfying the property

$$
\sum_{j, k \in \mathbb{Z}_{n}} W_{j k} \rho W_{j k}^{*}=n I_{H}, \rho \in \mathfrak{S}(H) .
$$

Quantum channels of the form

$$
\Phi(\rho)=\sum_{j, k \in \mathbb{Z}_{n}} \pi_{j k} W_{j k} \rho W_{j k}^{*}, \rho \in \mathfrak{S}(H)
$$

where $\left(\pi_{j k}\right)$ is a probability distribution, are said to be Weyl channels. Given a unitary representation $\lambda$ of $\mathbb{Z}_{n}$ in $H$ and a probability distribution $\left(p_{k}, k \in\right.$ $\mathbb{Z}_{n}$ ) a Weyl channel of the form

$$
\Psi_{\lambda}(\rho)=\sum_{k \in \mathbb{Z}_{n}} p_{k} \lambda(k) \rho \lambda(k)^{*}, \rho \in \mathfrak{S}(H)
$$

is said to be a phase damping channel.

Let us fix a phase damping channel of the form

$$
\Psi(\rho)=\sum_{k \in \mathbb{Z}_{n}} p_{k} V^{k} \rho V^{k *}, \rho \in \mathfrak{S}(H)
$$

where $p=\left(p_{k}, k \in \mathbb{Z}_{n}\right)$ is a probability distribution. Consider the quantum channel

$$
\Phi(\rho)=\frac{1}{n} \sum_{j \in \mathbb{Z}_{n}} U^{j} \Psi(\rho) U^{j *}=\frac{1}{n} \sum_{j, k \in \mathbb{Z}_{n}} p_{k} U^{j} V^{k} \rho V^{k *} U^{j *}, \rho \in \mathfrak{S}(H),
$$

Formula (6) gives a general form of the Weyl channel invariant with respect to the action of the group $\left(U^{j}, j \in \mathbb{Z}_{n}\right)$ in the sense

$$
U^{j} \Phi(\rho) U^{j *}=\Phi(\rho), \rho \in \mathfrak{S}(H), j \in \mathbb{Z}_{n} .
$$

It follows from (17) that

$$
\mathbb{E} \circ \Phi=\Phi,
$$

where the expectation $\mathbb{E}$ to the algebra of fixed elements with respect to the action of $\left(U^{j}, j \in \mathbb{Z}_{n}\right)$ is given by

$$
\mathbb{E}(\rho)=\frac{1}{n} \sum_{j \in \mathbb{Z}_{n}} U^{j} \rho U^{j *}, \rho \in \mathfrak{S}(H) .
$$


Put

$$
\Xi_{k}(\rho)=\frac{1}{n} \sum_{j \in \mathbb{Z}_{n}} U^{j} V^{k} \rho V^{k *} U^{j *}, \rho \in \mathfrak{S}(H), j \in \mathbb{Z}_{n},
$$

then (6) can be represented as

$$
\Phi(\rho)=\sum_{k \in \mathbb{Z}_{n}} p_{k} \Xi_{k}(\rho)
$$

The property (8) shows that $\Phi$ is a q-c channel and the additivity of $\bar{C}$ was shown in [1]. We place the following statement here to calculate the exact value of a classical capacity.

Proposition 1. Given a quantum channel $\Omega: \mathfrak{S}(K) \rightarrow \mathfrak{S}(K)$ and a pure state $|\xi\rangle\langle\xi| \in \mathfrak{S}(H \otimes K)$

$$
\inf _{\rho \in \mathfrak{S}(H \otimes K)} S(\Phi \otimes \Omega(|\xi\rangle\langle\xi|)) \geq-\sum_{k \in \mathbb{Z}_{n}} p_{k} \log p_{k}+S\left(\Omega\left(\operatorname{Tr}_{H}(|\xi\rangle\langle\xi|)\right)\right) .
$$

Proof.

Let us define a c-q channel $\Upsilon: \mathfrak{S}(H) \rightarrow \mathfrak{S}(H \otimes K)$ by the formula

$$
\Upsilon(\rho)=\sum_{k \in \mathbb{Z}_{n}}\left\langle e_{k}, \rho e_{k}\right\rangle\left(\Xi_{k} \otimes \Omega\right)(|\xi\rangle\langle\xi|), \rho \in \mathfrak{S}(H)
$$

Put

$$
\rho=\sum_{k \in \mathbb{Z}_{n}} p_{k}\left|e_{k}\right\rangle\left\langle e_{k}\right|, \sigma=\frac{1}{n} I_{H} .
$$

Applying the property of non-increasing the quantum relative entropy with respect to the action of quantum channel we obtain

$$
S(\Upsilon(\rho) \| \Upsilon(\sigma)) \leq S(\rho \| \sigma)
$$

It follows from (4) that

$$
\sum_{k \in \mathbb{Z}_{n}} \Xi_{k}(\rho)=I_{H}, \rho \in \mathfrak{S}(H) .
$$

Hence

$$
\sum_{k \in \mathbb{Z}_{n}}\left(\Xi_{k} \otimes \Omega\right)(|\xi\rangle\langle\xi|)=I_{H} \otimes \Omega\left(\operatorname{Tr}_{H}(|\xi\rangle\langle\xi|)\right)
$$

and

$$
\Upsilon(\sigma)=\frac{1}{n} I_{H} \otimes \Omega\left(\operatorname{Tr}_{H}(|\xi\rangle\langle\xi|)\right) .
$$


Substituting (9)-(11) to (10) we get

$$
\begin{gathered}
-S((\Phi \otimes \Omega)(|\xi\rangle\langle\xi|))-\operatorname{Tr}\left((\Phi \otimes \Omega)(|\xi\rangle\langle\xi|) \log \left(\frac{1}{n} I_{H} \otimes \Omega\left(\operatorname{Tr}_{H}(|\xi\rangle\langle\xi|)\right)\right)\right) \leq \\
\sum_{k \in \mathbb{Z}_{n}} p_{k} \log p_{k}-\operatorname{Tr}(\rho \log \sigma) .
\end{gathered}
$$

Taking into account that

$\operatorname{Tr}\left((\Phi \otimes \Omega)(|\xi\rangle\langle\xi|) \log \left(\frac{1}{n} I_{H} \otimes \Omega\left(\operatorname{Tr}_{H}(|\xi\rangle\langle\xi|)\right)\right)\right)=-\log n-S\left(\Omega\left(\operatorname{Tr}_{H}(|\xi\rangle\langle\xi|)\right)\right)$

and

$$
\operatorname{Tr}(\rho \log \sigma)=-\log n
$$

we obtain the result.

Corollary 1. Given a quantum channel $\Omega: \mathfrak{S}(K) \rightarrow \mathfrak{S}(K)$ and the $q-c$ Weyl channel (6) the following equality holds

$$
\inf _{\rho \in \mathfrak{S}(H \otimes K)} S((\Phi \otimes \Omega)(\rho))=\inf _{\rho \in \mathfrak{S}(H)} S(\Phi(\rho))+\inf _{\rho \in \mathfrak{S}(K)} S(\Omega(\rho)) .
$$

Proof.

Notice that

$$
S\left(\Phi\left(\left|e_{j}\right\rangle\left\langle e_{j}\right|\right)\right)=-\sum_{k \in \mathbb{Z}_{n}} p_{k} \log p_{k} \geq \inf _{\rho \in \mathfrak{S}(H)} S(\Phi(\rho))
$$

for any $j \in \mathbb{Z}_{n}$. It follows from Proposition 1 that

$$
\inf _{\rho \in \mathfrak{S}(H \otimes K)} S((\Phi \otimes \Omega)(\rho)) \geq \inf _{\rho \in \mathfrak{S}(H)} S(\Phi(\rho))+\inf _{\rho \in \mathfrak{S}(K)} S(\Omega(\rho)) .
$$

On the other hand, the right side in (12) can not be less than the left hand side. Hence,

$$
\inf _{\rho \in \mathfrak{S}(H)} S(\Phi(\rho))=-\sum_{k \in \mathbb{Z}_{n}} p_{k} \log p_{k}
$$

and we have the equality in (12).

Corollary 2. The classical capacity of the q-c Weyl channel (6) is given by the formula

$$
C(\Phi)=\log (n)+\sum_{k \in \mathbb{Z}_{n}} p_{k} \log p_{k}
$$


Proof.

The statement can be derived from the fact that

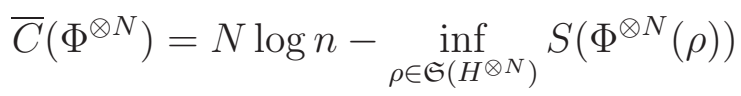

for covariant channels [20]. It follows from Corollary 1 that

$$
\inf _{\rho \in \mathfrak{S}\left(H^{\otimes N}\right)} S\left(\Phi^{\otimes N}(\rho)\right)=N \inf _{\rho \in \mathfrak{S}(H)} S(\Phi(\rho)) .
$$

In the proof of Corollary 1 we have shown that

$$
\inf _{\rho \in \mathfrak{S}(H)} S(\Phi(\rho))=-\sum_{k \in \mathbb{Z}_{n}} p_{k} \log p_{k}
$$

\section{Majorization}

Let $\mathfrak{J}$ be the index set and $|\mathfrak{J}|=d<+\infty$. Given a probability distribution $\lambda=\left(\lambda_{J}, J \in \mathfrak{J}\right)$ we denote $\lambda^{\downarrow}=\left(\lambda_{j}^{\downarrow}, 1 \leq j \leq d\right)$ the probability distribution obtained by sorting $\lambda$ in the decreasing order,

$$
\lambda_{1}^{\downarrow} \geq \lambda_{2}^{\downarrow} \geq \cdots \geq \lambda_{d}^{\downarrow}
$$

Consider two probability distribution $\lambda=\left(\lambda_{J}, J \in \mathfrak{J}\right)$ and $\mu=\left(\mu_{J}, J \in \mathfrak{J}\right)$. We shall say that $\lambda$ majorizes $\mu$ and write

$$
\mu \prec \lambda
$$

iff

$$
\sum_{j=1}^{k} \mu_{j}^{\downarrow} \leq \sum_{j=1}^{k} \lambda_{j}^{\downarrow}, \quad 1 \leq k \leq d .
$$

Let $H_{d}$ be a Hilbert space with $\operatorname{dim} H_{d}=d$. Denote $B\left(H_{d}\right)$ the algebra of all bounded operators in $H_{d}$. The following statement can be derived from [12] (see Theorem 2).

Proposition 2. Let $0 \leq X_{J} \leq I, J \in \mathfrak{J},|\mathfrak{J}|=d^{2}$, be a set of positive operators in $B\left(H_{d}\right)$ such that

$$
\sum_{J \in \mathfrak{J}} X_{J}=d I_{H_{d}}
$$


Then, given a probability distribution $\pi=\left(\pi_{J}, J \in \mathfrak{J}\right)$ the eigenvalues $\lambda=$ $\left(\lambda_{j}\right)_{j=1}^{d}$ of the positive operator

$$
A=\sum_{J \in \mathfrak{J}} \pi_{J} X_{J}
$$

sorted in the decreasing order $\lambda \equiv \lambda^{\downarrow}$ satisfy the relation

$$
\lambda \prec p,
$$

where

$$
p_{j}=\sum_{m=1+(j-1) d}^{d+(j-1) d} \pi_{m}^{\downarrow}, 1 \leq j \leq d .
$$

Proof.

Let $\left(e_{j}\right)_{j=1}^{d}$ be the unit eigenvectors corresponding to the eigenvalues $\left(\lambda_{j}\right)_{j=1}^{d}$. Then,

$$
\sum_{j=1}^{k} \lambda_{j}=\sum_{j=1}^{k}\left\langle e_{j}, A e_{j}\right\rangle=\sum_{j=1}^{k} \sum_{J \in \mathfrak{J}} \pi_{J}\left\langle e_{j}, X_{J} e_{j}\right\rangle \leq \sum_{j=1}^{k} p_{j}, 1 \leq k \leq d .
$$

Corollary 3. The eigenvalues $\lambda$ of the positive operator $A$ in Proposition 2 possess the property

$$
-\sum_{j=1}^{d} \lambda_{j} \log \lambda_{j} \geq-\sum_{j=1}^{d} p_{j} \log p_{j}
$$

Proof.

Since $\lambda$ majorizes $\mu$ due to Proposition 2, we get the result [21].

\section{Deformation of q-c Weyl channels}

Let us come back to Weyl channels (5).

Definition. Suppose that a probability distribution $\left(\pi_{j k}, j, k \in \mathbb{Z}_{n}\right)$ satisfies the relation

$$
\pi_{00} \geq \pi_{10} \geq \cdots \geq \pi_{n-10} \geq \pi_{01} \geq \ldots \pi_{n-11} \geq \pi_{02} \geq \cdots \geq \pi_{n-1 n-1} .
$$


Put

$$
p_{k}=\sum_{j \in \mathbb{Z}_{n}} \pi_{j k}, k \in \mathbb{Z}_{n}
$$

Then (5) is said to be the Weyl channel obtained by the deformation of q-c channel (6).

Theorem. The Weyl channel $\Phi$ obtained by the deformation of q-c channel satisfies the property

$$
\inf _{\rho \in \mathfrak{S}\left(H^{\otimes N}\right)} S\left(\Phi^{\otimes N}(\rho)\right)=-N \sum_{k=1}^{n} p_{j} \log p_{j} .
$$

Proof.

Denote $\mathfrak{J}$ the index set $\left(\mathbb{Z}_{n} \times \mathbb{Z}_{n}\right)^{\times N}$ consisting of collections $\left(j_{1}, k_{1}\right), \ldots,\left(j_{N}, k_{N}\right)$, $j_{s}, k_{s} \in \mathbb{Z}_{n}$. Let us consider the probability distribution $\Pi=\left(\Pi_{J}, J \in \mathfrak{J}\right)$ and a set of positive operators $\left(X_{J}, J \in \mathfrak{J}\right)$ defined by the formula

$$
\begin{gathered}
\Pi_{J}=\prod_{s=1}^{N} \pi_{j_{s} k_{s}}, \\
X_{J}=\left(\otimes_{s=1}^{N} W_{j_{s} k_{s}}\right) \rho\left(\otimes_{s=1}^{N} W_{j_{s} k_{s}}^{*}\right), J \in \mathfrak{J},
\end{gathered}
$$

where $\rho$ is a fixed state in $\mathfrak{S}\left(H^{\otimes N}\right)$. Then, the conditions of Proposition 2 is satisfied for $\left(\Pi_{J}\right),\left(X_{J}\right)$ and $d=n^{N}$. Applying Corollary 3 we obtain

$$
S(\Phi(\rho)) \geq-N \sum_{j=1}^{N} p_{j} \log p_{j} .
$$

The equality in (16) is achieved for any

$$
\rho=|e\rangle\langle e|
$$

where

$$
e=\otimes_{s=1}^{N} e_{j_{s}}, j_{s} \in \mathbb{Z}_{n}
$$

Corollary 4. The classical capacity of the Weyl channel obtained by the deformation of (6) is given by the formula

$$
C(\Phi)=\log (n)+\sum_{k \in \mathbb{Z}_{n}} p_{k} \log p_{k} .
$$

Proof. 
The statement can be derived from the fact that

$$
\bar{C}\left(\Phi^{\otimes N}\right)=N \log n-\inf _{\rho \in \mathfrak{S}(H \otimes N)} S\left(\Phi^{\otimes N}(\rho)\right)
$$

for covariant channels [20]. It follows from Theorem that

$$
\inf _{\rho \in \mathfrak{S}(H \otimes N)} S\left(\Phi^{\otimes N}(\rho)\right)=N \inf _{\rho \in \mathfrak{S}(H)} S(\Phi(\rho))=-N \sum_{j=1}^{N} p_{j} \log p_{j} .
$$

\subsection{Example: qutrits}

Because the qubit case $\operatorname{dim} H=2$ is completely parsed [4] a simplest example of the introduced techniques can be given for qutrits, $\operatorname{dim} H=3$. Let us define two unitary operators $U$ and $V$ satisfying (3)

$$
\begin{gathered}
U e_{0}=e_{0}, U e_{1}=e^{i \frac{2 \pi}{3}} e_{1}, U e_{2}=e^{i \frac{4 \pi}{3}} e_{2}, \\
V e_{0}=e_{1}, V e_{1}=e_{2}, V e_{2}=e_{0} .
\end{gathered}
$$

Then, consider the expectation (86)

$$
\mathbb{E}(x)=\frac{1}{3} \sum_{j=0}^{2} U^{j} x U^{j *}, x \in B(H) .
$$

Taking a probability distribution $\left\{p_{0}, p_{1}, p_{2}\right\}$ we can define a qc Weyl channel by the formula

$$
\Phi_{q c}(\rho)=\mathbb{E} \circ \sum_{k=0}^{2} p_{k} V^{k} \rho V^{k *}, \rho \in \mathfrak{S}(H) .
$$

It follows from Corollary 1 and Corollary 2 that

$$
\inf _{\rho \in \mathfrak{S}(H \otimes K)} S\left(\left(\Phi_{q c} \otimes \Omega\right)(\rho)\right)=\inf _{\rho \in \mathfrak{S}(H)} S\left(\Phi_{q c}(\rho)\right)+\inf _{\rho \in \mathfrak{S}(K)} S(\Omega(\rho))
$$

for any quantum channel $\Omega: \mathfrak{S}(K) \rightarrow \mathfrak{S}(K)$ and the classical capacity is equal to

$$
C\left(\Phi_{q c}\right)=n+\sum_{k=0}^{2} p_{k} \log p_{k}
$$


Suppose that $p_{0} \geq p_{1} \geq p_{2}$ and one can pick up positive numbers $\pi_{j k}, 0 \leq$ $j, k \leq 2$, satisfying the relations

$$
\begin{gathered}
\pi_{00} \geq \pi_{10} \geq \pi_{20} \geq \pi_{01} \geq \pi_{11} \geq \pi_{21} \geq \pi_{02} \geq \pi_{12} \geq \pi_{22} \\
p_{k}=\pi_{0 k}+\pi_{1 k}+\pi_{2 k}, 0 \leq k \leq 2
\end{gathered}
$$

Then,

$$
\Phi(\rho)=\sum_{j, k=0}^{2} \pi_{j k} U^{j} V^{k} \rho V^{k *} U^{j *}, \rho \in \mathfrak{S}(H),
$$

is the Weyl channel obtained by the deformation of (17). Applying Corollary 4 we obtain for a classical capacity

$$
C(\Phi)=\log (3)+p_{0} \log p_{0}+p_{1} \log p_{1}+p_{2} \log p_{2} .
$$

As a concrete example one can take

$$
p_{0}=\frac{1}{2}, p_{1}=\frac{1}{3}, p_{2}=\frac{1}{6} .
$$

In the case, one of possible deformations is given by

$$
\begin{gathered}
\pi_{00}=\frac{1}{4}, \pi_{10}=\frac{1}{8}, \pi_{20}=\frac{1}{8}, \\
\pi_{01}=\frac{1}{8}, \pi_{11}=\frac{1}{8}, \pi_{21}=\frac{1}{12} \\
\pi_{02}=\frac{1}{12}, \pi_{12}=\frac{1}{24}, \pi_{22}=\frac{1}{24} .
\end{gathered}
$$

\section{Acknowledgments}

The author is grateful to A.S. Holevo for fruitful discussion and useful comments.

\section{References}

[1] A. S. Holevo, The capacity of the quantum channel with general signal states, IEEE Trans. Inform. Theory, 44:1 (1998), 269-273

[2] B. Schumacher, M.D. Westmoreland, Sending classical information via noisy quantum channels, Physical Review A 56 (1997) 131 
[3] G. G. Amosov, A. S. Holevo, R. F. Werner, On the Additivity Conjecture in Quantum Information Theory, Problems Inform. Transmission, 36:4 (2000), 305-313

[4] C. King, Additivity for unital qubit channels, J. Math. Phys. 43 (2002) 4641-4653

[5] C. King, The capacity of the quantum depolarizing channel, IEEE Trans. Inform. Theory 49 (2003) 221-229

[6] P. Shor, Additivity of the classical capacity of entanglement-breaking quantum channels, J. Math. Phys. 43 (2002) 4334-4340

[7] A. S. Holevo, Complementary channels and the additivity problem, Theory Probab. Appl., 51:1 (2007), 92-100

[8] N. Datta, M. Fukuda, A. S. Holevo, Complementarity and additivity for covariant channels, Quantum Inf. Process., 5:3 (2006), 179-207

[9] V. Giovannetti, R. Garcia-Patron, N. J. Cerf, A. S. Holevo, Ultimate classical communication rates of quantum optical channels, Nature Photonics, 8:10 (2014), 216, 6 pp.

[10] A. Mari, V. Giovannetti, A. S. Holevo, Quantum state majorization at the output of bosonic Gaussian channels, Nature Communications, 5 (2014), 3826, 5 pp.

[11] M.B. Hastings, A Counterexample to additivity of minimum output entropy, Nature Physics 5 (2009) 255-257

[12] J. ur Rehman, Y. Jeong, J.S. Kim, H. Shin, Holevo Capacity of Discrete Weyl Channels, arXiv:2003.01942

[13] G. Lindblad, Completely positive maps and entropy inequalities, Comm. Math. Phys. 40 (1975) 147-151. 1975

[14] G. G. Amosov, On Weyl channels being covariant with respect to the maximum commutative group of unitaries, J. Math. Phys., 48:1 (2007), 012104, 14 pp.

[15] G. G. Amosov, Remark on the Additivity Conjecture for a Quantum Depolarizing Channel, Problems Inform. Transmission, 42:2 (2006), 69-76

[16] G. G. Amosov, Strong superadditivity conjecture holds for the quantum depolarizing channel in any dimension, Phys. Rev. A, 75:6 (2007), 060304(R), 2 pp. 
[17] G. G. Amosov, S. Mancini, The decreasing property of relative entropy and the strong superadditivity of quantum channels, Quantum Inf. Comput., 9:7-8 (2009), 594-609

[18] G. G. Amosov, On estimating the output entropy of the tensor product of a phase-damping channel and an arbitrary channel, Problems Inform. Transmission, 49:3 (2013), 224-231

[19] G. G. Amosov, Estimating the output entropy of a tensor product of two quantum channels, Theoret. and Math. Phys., 182:3 (2015), 397-406

[20] A. S. Holevo, On the constrained classical capacity of infinitedimensional covariant quantum channels, J. Math. Phys., 57:1 (2016), 15203,11 pp.

[21] R. Bhatia, Matrix analysis, Springer 1996. 